\title{
Efficient Carrier Multiplication in Low Band Gap Mixed Sn/Pb Halide Perovskites
}

\author{
Sourav Maiti, Silvia Ferro, Deepika Poonia, Bruno Ehrler, Sachin Kinge, and Laurens D. A. Siebbeles* \\ Cite This: J. Phys. Chem. Lett. 2020, 11, 6146-6149 \\ Read Online
}

ABSTRACT: Carrier multiplication (CM) generates multiple electron-hole pairs in a semiconductor from a single absorbed photon with energy exceeding twice the band gap. Thus, CM provides a promising way to circumvent the ShockleyQueisser limit of solar cells. The ideal material for CM should have significant overlap with the solar spectrum and should be able to fully utilize the excess energy above the band gap for additional charge carrier generation. We report efficient $\mathrm{CM}$ in mixed $\mathrm{Sn} / \mathrm{Pb}$ halide perovskites (band gap of $1.28 \mathrm{eV}$ ) with onset just above twice the band gap. The CM rate outcompetes the carrier cooling process leading to efficient $\mathrm{CM}$ with a quantum yield of 2 for photoexcitation at 2.8 times the band gap. Such efficient CM characteristics add to the many advantageous properties of mixed $\mathrm{Sn} / \mathrm{Pb}$ metal halide perovskites for photovoltaic applications.

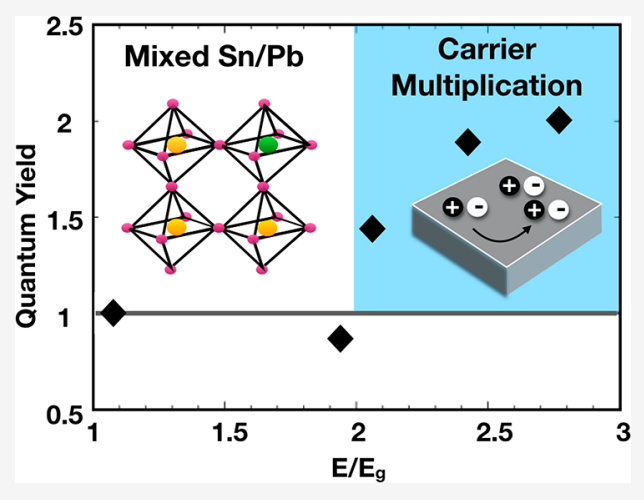

$\mathrm{C}$ arrier multiplication (CM) is a process of generating multiple electron-hole pairs in a material by absorption of one photon. This process is of particular interest to increase the efficiency of photovoltaic devices. In conventional solar cells the excess energy of photons higher than the band gap $\left(E_{\mathrm{g}}\right)$ of a semiconductor is wasted as heat because of rapid thermalization of the charge carriers. In CM, the excess energy of an electron and/or a hole higher than the band gap is utilized to create additional charge carriers. In this way, the photovoltaic efficiency can surpass the Shockley-Queisser limit of 33\% for singlejunction solar cells. ${ }^{1-7}$ The exploitation of CM in such solar cells is optimal for semiconductors with a band gap in the nearinfrared (NIR). ${ }^{2} \mathrm{CM}$ has already been established for $\mathrm{Pb}$ chalcogenide materials with different degrees of quantum confinement. $^{8-13}$ However, most of these materials suffer from a high onset energy of CM and/or a low CM efficiency.

Recently, efficient CM with onset around twice the band gap and 2.25 times the band gap has been reported for $\mathrm{CsPbI}_{3}$ and $\mathrm{FAPbI}_{3}$ perovskite nanocrystals, respectively. ${ }^{14-16}$ However, these perovskites have a band gap in the visible wavelength region and are therefore impractical for solar cell applications. The band gap is reduced to the NIR region in mixed $\mathrm{Sn} / \mathrm{Pb}$ halide perovskites, making them promising for application in solar cells that exploit CM. ${ }^{17-23}$ Moreover, the low exciton binding energy $(\sim 16 \mathrm{meV})$ in these perovskites ensures that photoexcitation results in mostly free charges that can be easily extracted. ${ }^{19}$ Here we present a study on $\mathrm{CM}$ in a mixed $\mathrm{Sn} / \mathrm{Pb}$ halide perovskite of band gap $1.28 \mathrm{eV}$ through ultrafast differential transient absorption (TA) spectroscopy. For such a band gap, theoretically, CM can enhance the efficiency of a solar cell from the Shockley-Queisser limit to $40 \%{ }^{24}$ Our experimental results show that the onset of $\mathrm{CM}$ is close to twice the band gap in this perovskite. At higher photon energies multiple electron-hole pairs are produced for each absorbed photon with the generation of 2 electron-hole pairs at photon energy 2.8 times the band gap.

A mixed $\mathrm{Sn} / \mathrm{Pb}$ low band gap perovskite with the composition $\left(\mathrm{FASnI}_{3}\right)_{0.6}\left(\mathrm{MAPbI}_{3}\right)_{0.4}$ was synthesized following a reported procedure with small modifications. ${ }^{22}$ The details of the synthesis are provided in the experimental section of the Supporting Information. The scanning electron microscope (SEM) images (Figure S1) of the perovskite film reveal compact grain boundaries, average grain size of about $1 \mu \mathrm{m}$, and a low density of pinholes.

Figure 1 shows the fraction absorbed light, $F_{\mathrm{A}}$, of the thin film sample as a function of photon energy. The absorption spectrum exhibits the shoulder of an absorption peak near $1.3 \mathrm{eV}$. The band gap was determined to be $1.28 \mathrm{eV}$ from the Tauc plot shown in the inset of Figure 1.

The photogeneration and decay dynamics of electron-hole pairs were studied by time-resolved optical pump-probe measurements, as detailed before. ${ }^{25,26}$ A laser pump pulse produces electron-hole pairs that subsequently undergo energetic relaxation (cooling) to the band edge and eventually decay by recombination. The charge carriers cause a differential change in absorption of the probe pulse given by

Received: June 9, 2020

Accepted: July 16, 2020

Published: July 16, 2020 


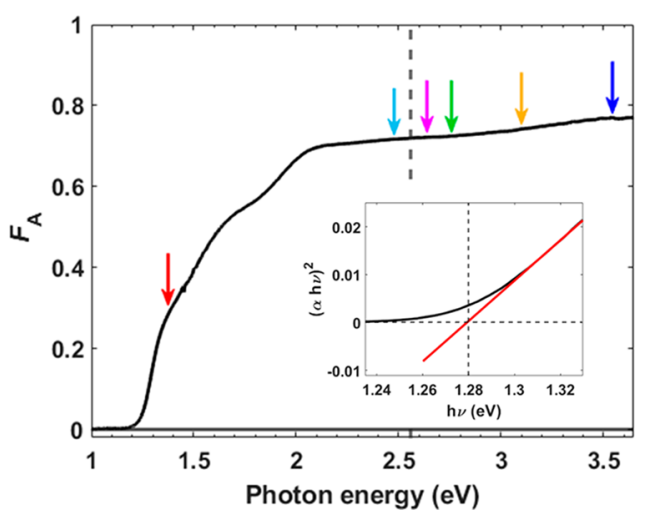

Figure 1. Steady-state optical absorption spectrum of the $\mathrm{Sn} / \mathrm{Pb}$ halide perovskite film. The vertical dotted line represents twice the band gap energy. The colored arrows indicate the photon energies at which the quantum yield of electron-hole pairs was determined. The inset shows a Tauc plot, which is used to determine the band gap.

$$
\Delta A=\log _{10}\left(\frac{I_{\text {off }}^{\text {probe }}}{I_{\text {on }}^{\text {probe }}}\right)
$$

where $I_{\text {off }}^{\text {probe }}$ and $I_{\text {on }}^{\text {probe }}$ are the transmitted probe pulse intensities without and with a pump laser pulse, respectively. Note that $\Delta A$ corresponds to the change in the optical density. The photogeneration quantum yield (QY) of electron-hole pairs $(\varphi)$ (i.e., the number of electron-hole pairs produced per absorbed pump photon) can be obtained from the relation $\frac{|\Delta A|}{I_{0} F_{A}}=\varphi \frac{\sigma_{B}}{\ln 10}$. Here, $I_{0}$ is the incident number of pump photons per unit area and $\sigma_{\mathrm{B}}$ the cross section for induced absorption or bleach of a probe photon due to the presence of an electronhole pair.

Figure 2 shows $\Delta A / I_{0} F_{\mathrm{A}}$ for a photoexcitation energy of 1.38 $\mathrm{eV}$, which is close to the band gap where $\mathrm{CM}$ is not possible, and

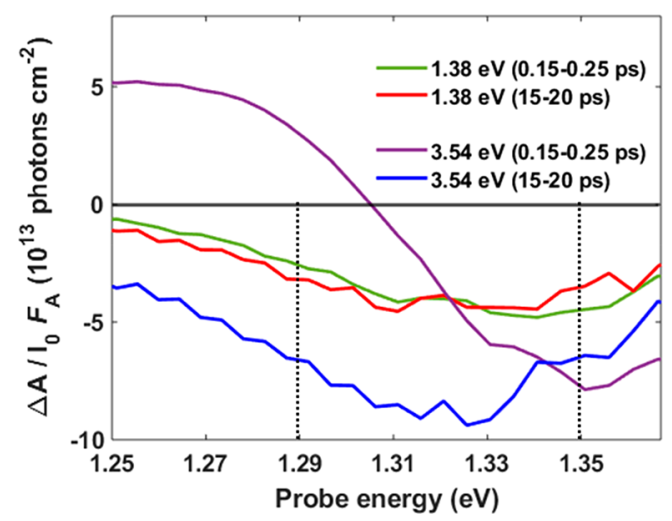

Figure 2. Transient change in optical absorption normalized to the absorbed density of pump photons, $\Delta A / I_{0} F_{\mathrm{A}}$, upon photoexcitation at 1.38 and $3.54 \mathrm{eV}$ for two different pump-probe time delays, as indicated. The dotted vertical lines mark the spectral region used for $\mathrm{CM}$ analysis.

for $3.54 \mathrm{eV}$ which is more than twice the band gap so that $\mathrm{CM}$ can occur. For $1.38 \mathrm{eV}$, the spectrum exhibits a ground-state bleach due to depletion of the electron population in the valence band. The spectral shape for $1.38 \mathrm{eV}$ does not exhibit a change over time, because the electron-hole pairs are generated close to the band gap and do not undergo significant cooling. For photoexcitation at the higher energy of $3.54 \mathrm{eV}$, the shape of the
TA spectrum shows a large change with time going from 0.15 to 0.25 ps to $15-20$ ps. Excitation at $3.54 \mathrm{eV}$ initially creates hot charges, leading to a photoinduced absorption (PIA) feature present on the lower-energy side of the spectrum. In similar perovskite structures, this PIA has been attributed to band gap renormalization by hot carriers. ${ }^{27,28}$ As the carriers dissipate excess energy and cool to the band edge, the PIA turns into bleach, as can be seen in Figure 2 for $15-20$ ps delay time. The presence of hot carriers can change the shape of the spectra, and the magnitude of the TA can be higher for a hot electron-hole pair compared to a cold electron-hole pair. ${ }^{29}$ Therefore, for further analysis, we used TA spectra at time delays between 15 and $20 \mathrm{ps}$, because then the shape of the spectra is independent of photoexcitation energy (see Figure S2), indicating that the charge carriers have completed cooling to the band edge. This assignment is further corroborated by similar decay kinetics of the TA bleach after 10 ps for photoexcitation at both 1.38 and $3.54 \mathrm{eV}$ (see Figure S3). Hence, the higher amplitude of $\Delta A$ / $I_{0} F_{\mathrm{A}}$ at $15-20 \mathrm{ps}$ delay time in Figure 2 for $3.54 \mathrm{eV}$ excitation compared to $1.38 \mathrm{eV}$ excitation indicates an increase of the $\mathrm{QY}$ of electron-hole pairs.

To further quantify the CM efficiency, we averaged the magnitude of the TA at probe energies from 1.29 to $1.35 \mathrm{eV}$ in the time range of $15-20$ ps. The average magnitude of $|\Delta A|$ is plotted against the number of absorbed photon fluence in Figure 3. The linear increase of $|\Delta A|$ with absorbed pump fluence for all

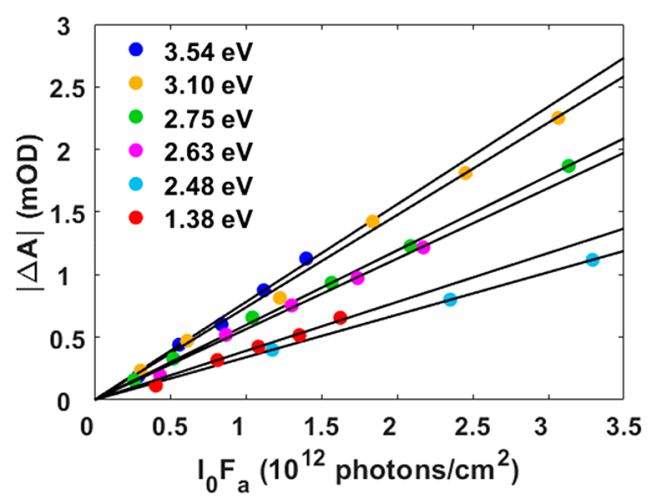

Figure 3. $|\Delta A|$ as a function of absorbed photons per unit area for different photoexcitation energies. The solid lines are a linear fit to the data. The increase in the slope with pump photon energy reflects a higher QY due to CM. Error bars (standard deviations) for representative excitation energies are shown in Figure S5.

photoexcitation energies excludes the occurrence of decay of electron-hole pairs by higher-order recombination. Moreover, the bleach decay kinetics for $3.54 \mathrm{eV}$ excitation for the lowest $\left(0.3 \times 10^{12}\right.$ photons $\left./ \mathrm{cm}^{2}\right)$ and highest $\left(1.4 \times 10^{12}\right.$ photons/ $\mathrm{cm}^{2}$ ) fluence used in the experiments are similar (Figure S4), which further demonstrates the absence of higher-order decay. For each photoexcitation energy in Figure 3, the fluence dependence of $|\Delta A|$ was linearly fit to obtain the slope, which is directly proportional to the QY, $\varphi$, as discussed above. For photoexcitation close to the band gap $(1.38 \mathrm{eV})$, the $\mathrm{QY}$ is taken as unity. The relative $\mathrm{QY}$ at all other photoexcitation energies is calculated as the ratio of the slopes at the respective photoexcitation energy to the slope at $1.38 \mathrm{eV}$ excitation energy.

In Figure 4 the QY is plotted as a function of photoexcitation energy ( $E$, top axis) and band gap multiple ( $E / E_{\mathrm{g}}$, bottom axis). The error bars represent the $95 \%$ confidence interval of the slope resulting from the linear fit. The QY should remain 1 for 


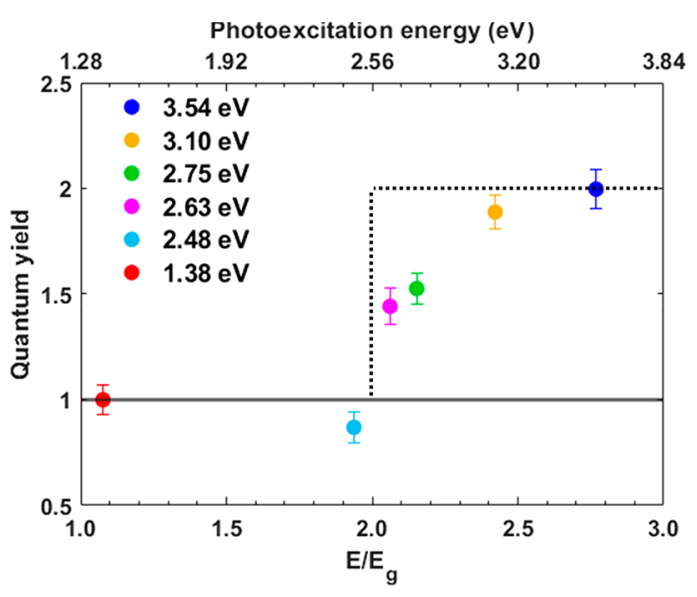

Figure 4. Variation of the QY with photoexcitation energy (top axis) and with band gap multiple ( $E / E_{\mathrm{g}}$, bottom axis).

photoexcitation energy below twice the band gap $\left(E_{\mathrm{g}} \leq E<2 E_{\mathrm{g}}\right)$ which is indeed the case within the experimental noise.

The onset of CM is about twice the band gap, $E_{\mathrm{g}}$, and the QY gradually increases to a value of 2 for $E / E_{\mathrm{g}}$ equal to 2.8 . These results are quite interesting compared to other materials, especially $\mathrm{Pb}$-chalcogenides and $\mathrm{Pb}$-based perovskites where $\mathrm{CM}$ has been extensively investigated. For a percolative network of interconnected $\mathrm{PbSe} \mathrm{NCs}$, the $\mathrm{CM}$ onset was around twice the band gap; however, the $\mathrm{QY}$ was lower than 2 for photoexcitation energies up to 4 times the band gap. ${ }^{24} \mathrm{CsPbI}_{3}$ perovskite nanocrystals have $\mathrm{CM}$ onset just above twice the band gap; however, the QY is only 1.6 near 2.5 times the band gap (Figure S6). ${ }^{14}$ Recently, CM with onset and efficiency similar to the low band gap perovskites of the present study has been reported for $\mathrm{MoTe}_{2}$ and $\mathrm{WSe}_{2}$ (see Figure S6 for comparison), although the explanation in these materials remains elusive. ${ }^{30}$ In $\mathrm{Pb}$-chalcogenides, the excess energy above the band gap is equally distributed between electron and hole, which sets the CM threshold at three times the band gap. If the photon energy in excess of the band gap is taken up completely by one of the charge carriers and utilized for CM then, ideally, the QY can reach 2 at exactly twice the band gap. Such asymmetric distribution is possible involving an optical transition from the highest valence band maximum (second valence band maximum) to a second conduction band (lowest conduction band), so that all excess photon energy is transferred to the electron (hole). ${ }^{31}$ Thus, the CM threshold can be near twice the band gap if this second conduction (valence) band has close to twice the band gap energy, as has been proposed for the percolative network of PbSe NCs. ${ }^{24}$ Therefore, we speculate the $\mathrm{CM}$ onset near twice the band gap in the current low band gap perovskites may result from an asymmetric distribution of excess energy between the electron and the hole due to the presence of a second conduction (valence) band close to twice the band gap. $^{31,32}$ In agreement with the reasoning above, for $\mathrm{Pb}$ chalcogenide bulk and quantum dots, the CM threshold was found to correspond to asymmetric transitions involving a second conduction (valence) band. This suggests quantum confinement may not be required for efficient $\mathrm{CM} .{ }^{31} \mathrm{~A}$ recent theoretical study by Guan et al. suggests the presence of a second conduction band at 2.2 times the band gap. ${ }^{33}$ Note that the band gap obtained through density functional theory (DFT) in that paper is lower than the experimental band gap. More advanced band structure calculations, e.g. by the GW-method, are required to get further insight into the possibility of an asymmetric transition.

In conclusion, we have shown efficient $\mathrm{CM}$ in a bulk $\mathrm{Sn} / \mathrm{Pb}$ halide perovskite with an onset for photoexcitation energy near twice the band gap and QY reaching 2 at around 2.8 times the band gap. This result for a bulk material shows that quantum confinement is not a strict requirement for CM. As compared to nanostructures, a bulk material typically has the advantage of more efficient charge transport to external electrodes in a photovoltaic device. The occurrence of efficient CM in combination with the prospect of efficient charge transport makes mixed $\mathrm{Sn} / \mathrm{Pb}$ halide perovskites promising candidates for multiexcitonic solar cell applications.

\section{ASSOCIATED CONTENT}

\section{Supporting Information}

The Supporting Information is available free of charge at https://pubs.acs.org/doi/10.1021/acs.jpclett.0c01788.

Characterization, transient spectra for all excitation energies, and kinetic traces (PDF)

\section{AUTHOR INFORMATION}

\section{Corresponding Author}

Laurens D. A. Siebbeles - Optoelectronic Materials Section, Department of Chemical Engineering, Delft University of Technology, Delft 2629 HZ, The Netherlands; (1) orcid.org/ 0000-0002-4812-7495; Email: 1.d.a.siebbeles@tudelft.nl

\section{Authors}

Sourav Maiti - Optoelectronic Materials Section, Department of Chemical Engineering, Delft University of Technology, Delft 2629 HZ, The Netherlands; (1) orcid.org/0000-0003-1983-9159

Silvia Ferro - Center for Nanophotonics, AMOLF, Amsterdam, The Netherlands

Deepika Poonia - Optoelectronic Materials Section, Department of Chemical Engineering, Delft University of Technology, Delft $2629 \mathrm{HZ}$, The Netherlands

Bruno Ehrler - Center for Nanophotonics, AMOLF, Amsterdam, The Netherlands; 이이이.org/0000-0002-5307-3241

Sachin Kinge - Optoelectronic Materials Section, Department of Chemical Engineering, Delft University of Technology, Delft 2629 HZ, The Netherlands; Materials Research \& Development, Toyota Motor Europe, B-1913 Zaventem, Belgium

Complete contact information is available at:

https://pubs.acs.org/10.1021/acs.jpclett.0c01788

\section{Notes}

The authors declare no competing financial interest.

\section{ACKNOWLEDGMENTS}

This research received funding from The Netherlands Organisation for Scientific Research (NWO) in the framework of the Materials for sustainability and from the Ministry of Economic Affairs in the framework of the PPP allowance.

\section{REFERENCES}

(1) Shockley, W.; Queisser, H. J. Detailed Balance Limit of Efficiency of p-n Junction Solar Cells. J. Appl. Phys. 1961, 32 (3), 510-519.

(2) Hanna, M. C.; Nozik, A. J. Solar Conversion Efficiency of Photovoltaic and Photoelectrolysis Cells with Carrier Multiplication Absorbers. J. Appl. Phys. 2006, 100 (7), 074510.

(3) Beard, M. C.; Midgett, A. G.; Hanna, M. C.; Luther, J. M.; Hughes, B. K.; Nozik, A. J. Comparing Multiple Exciton Generation in Quantum 
Dots To Impact Ionization in Bulk Semiconductors: Implications for Enhancement of Solar Energy Conversion. Nano Lett. 2010, 10 (8), 3019-3027.

(4) Beard, M. C.; Luther, J. M.; Semonin, O. E.; Nozik, A. J. Third Generation Photovoltaics based on Multiple Exciton Generation in Quantum Confined Semiconductors. Acc. Chem. Res. 2013, 46 (6), $1252-1260$.

(5) Smith, C.; Binks, D. Multiple Exciton Generation in Colloidal Nanocrystals. Nanomaterials 2014, 4 (1), 19-45.

(6) ten Cate, S.; Sandeep, C. S. S.; Liu, Y.; Law, M.; Kinge, S.; Houtepen, A. J.; Schins, J. M.; Siebbeles, L. D. A. Generating Free Charges by Carrier Multiplication in Quantum Dots for Highly Efficient Photovoltaics. Acc. Chem. Res. 2015, 48 (2), 174-181.

(7) Davis, N. J. L. K.; Böhm, M. L.; Tabachnyk, M.; WisniveskyRocca-Rivarola, F.; Jellicoe, T. C.; Ducati, C.; Ehrler, B.; Greenham, N. C. Multiple-Exciton Generation in Lead Selenide Nanorod Solar Cells with External Quantum Efficiencies Exceeding 120\%. Nat. Commun. 2015, 6 (1), 8259.

(8) Ellingson, R. J.; Beard, M. C.; Johnson, J. C.; Yu, P.; Micic, O. I.; Nozik, A. J.; Shabaev, A.; Efros, A. L. Highly Efficient Multiple Exciton Generation in Colloidal PbSe and PbS Quantum Dots. Nano Lett. 2005, 5 (5), 865-871.

(9) Trinh, M. T.; Houtepen, A. J.; Schins, J. M.; Hanrath, T.; Piris, J.; Knulst, W.; Goossens, A. P.; Siebbeles, L. D. In spite of Recent Doubts Carrier Multiplication Does Occur in PbSe Nanocrystals. Nano Lett. 2008, 8 (6), 1713-8.

(10) Aerts, M.; Suchand Sandeep, C. S.; Gao, Y.; Savenije, T. J.; Schins, J. M.; Houtepen, A. J.; Kinge, S.; Siebbeles, L. D. A. Free Charges Produced by Carrier Multiplication in Strongly Coupled PbSe Quantum Dot Films. Nano Lett. 2011, 11 (10), 4485-4489.

(11) Padilha, L. A.; Stewart, J. T.; Sandberg, R. L.; Bae, W. K.; Koh, W.-K.; Pietryga, J. M.; Klimov, V. I. Carrier Multiplication in Semiconductor Nanocrystals: Influence of Size, Shape, and Composition. Acc. Chem. Res. 2013, 46 (6), 1261-1269.

(12) Kershaw, S. V.; Rogach, A. L. Carrier Multiplication Mechanisms and Competing Processes in Colloidal Semiconductor Nanostructures. Materials 2017, 10 (9), 1095.

(13) Aerts, M.; Bielewicz, T.; Klinke, C.; Grozema, F. C.; Houtepen, A. J.; Schins, J. M.; Siebbeles, L. D. A. Highly Efficient Carrier Multiplication in PbS nanosheets. Nat. Commun. 2014, 5 (1), 3789.

(14) de Weerd, C.; Gomez, L.; Capretti, A.; Lebrun, D. M.; Matsubara, E.; Lin, J.; Ashida, M.; Spoor, F. C. M.; Siebbeles, L. D. A.; Houtepen, A. J.; et al. Efficient Carrier Multiplication in CsPbI3 Perovskite Nanocrystals. Nat. Commun. 2018, 9 (1), 4199.

(15) Cong, M.; Yang, B.; Chen, J.; Hong, F.; Yang, S.; Deng, W.; Han, K. Carrier Multiplication and Hot-Carrier Cooling Dynamics in Quantum-Confined CsPbI3 Perovskite Nanocrystals. J. Phys. Chem. Lett. 2020, 11 (5), 1921-1926.

(16) Li, M.; Begum, R.; Fu, J.; Xu, Q.; Koh, T. M.; Veldhuis, S. A.; Grätzel, M.; Mathews, N.; Mhaisalkar, S.; Sum, T. C. Low Threshold and Efficient Multiple Exciton Generation in Halide Perovskite Nanocrystals. Nat. Commun. 2018, 9 (1), 4197.

(17) Stoumpos, C. C.; Malliakas, C. D.; Kanatzidis, M. G. Semiconducting Tin and Lead Iodide Perovskites with Organic Cations: Phase Transitions, High Mobilities, and Near-Infrared Photoluminescent Properties. Inorg. Chem. 2013, 52 (15), 9019-9038.

(18) Hao, F.; Stoumpos, C. C.; Chang, R. P. H.; Kanatzidis, M. G. Anomalous Band Gap Behavior in Mixed $\mathrm{Sn}$ and $\mathrm{Pb}$ Perovskites Enables Broadening of Absorption Spectrum in Solar Cells. J. Am. Chem. Soc. 2014, 136 (22), 8094-8099.

(19) Galkowski, K.; Surrente, A.; Baranowski, M.; Zhao, B.; Yang, Z.; Sadhanala, A.; Mackowski, S.; Stranks, S. D.; Plochocka, P. Excitonic Properties of Low-Band-Gap Lead-Tin Halide Perovskites. ACS Energy Lett. 2019, 4 (3), 615-621.

(20) Eperon, G. E.; Leijtens, T.; Bush, K. A.; Prasanna, R.; Green, T.; Wang, J. T.-W.; McMeekin, D. P.; Volonakis, G.; Milot, R. L.; May, R.; et al. Perovskite-Perovskite Tandem Photovoltaics with Optimized Band Gaps. Science 2016, 354 (6314), 861.
(21) Zhao, D.; Yu, Y.; Wang, C.; Liao, W.; Shrestha, N.; Grice, C. R.; Cimaroli, A. J.; Guan, L.; Ellingson, R. J.; Zhu, K.; et al. Low-Bandgap Mixed Tin-Lead Iodide Perovskite Absorbers with Long Carrier Lifetimes for All-Perovskite Tandem Solar Cells. Nat. Energy 2017, 2 (4), 17018.

(22) Tong, J.; Song, Z.; Kim, D. H.; Chen, X.; Chen, C.; Palmstrom, A. F.; Ndione, P. F.; Reese, M. O.; Dunfield, S. P.; Reid, O. G.; et al. Carrier Lifetimes of $>1 \mu \mathrm{s}$ in Sn-Pb Perovskites Enable Efficient All-Perovskite Tandem Solar Cells. Science 2019, 364 (6439), 475.

(23) Bowman, A. R.; Klug, M. T.; Doherty, T. A. S.; Farrar, M. D.; Senanayak, S. P.; Wenger, B.; Divitini, G.; Booker, E. P.; AndajiGarmaroudi, Z.; Macpherson, S.; et al. Microsecond Carrier Lifetimes, Controlled p-Doping, and Enhanced Air Stability in Low-Bandgap Metal Halide Perovskites. ACS Energy Lett. 2019, 4 (9), 2301-2307.

(24) Kulkarni, A.; Evers, W. H.; Tomíc, S.; Beard, M. C.; Vanmaekelbergh, D.; Siebbeles, L. D. A. Efficient Steplike Carrier Multiplication in Percolative Networks of Epitaxially Connected $\mathrm{PbSe}$ Nanocrystals. ACS Nano 2018, 12 (1), 378-384.

(25) Spoor, F. C. M.; Kunneman, L. T.; Evers, W. H.; Renaud, N.; Grozema, F. C.; Houtepen, A. J.; Siebbeles, L. D. A. Hole Cooling Is Much Faster than Electron Cooling in PbSe Quantum Dots. ACS Nano 2016, 10 (1), 695-703.

(26) Spoor, F. C. M.; Tomić, S.; Houtepen, A. J.; Siebbeles, L. D. A. Broadband Cooling Spectra of Hot Electrons and Holes in PbSe Quantum Dots. ACS Nano 2017, 11 (6), 6286-6294.

(27) Price, M. B.; Butkus, J.; Jellicoe, T. C.; Sadhanala, A.; Briane, A.; Halpert, J. E.; Broch, K.; Hodgkiss, J. M.; Friend, R. H.; Deschler, F. Hot-Carrier Cooling and Photoinduced Refractive Index Changes in Organic-Inorganic Lead Halide Perovskites. Nat. Commun. 2015, 6 (1), 8420.

(28) Verma, S. D.; Gu, Q.; Sadhanala, A.; Venugopalan, V.; Rao, A. Slow Carrier Cooling in Hybrid $\mathrm{Pb}-\mathrm{Sn}$ Halide Perovskites. ACS Energy Lett. 2019, 4 (3), 736-740.

(29) Schmitt-Rink, S.; Chemla, D. S.; Miller, D. A. B. Theory of Transient Excitonic Optical Nonlinearities in Semiconductor Quantum-Well Structures. Phys. Rev. B: Condens. Matter Mater. Phys. 1985, 32 (10), 6601-6609.

(30) Kim, J.-H.; Bergren, M. R.; Park, J. C.; Adhikari, S.; Lorke, M.; Frauenheim, T.; Choe, D.-H.; Kim, B.; Choi, H.; Gregorkiewicz, T.; et al. Carrier Multiplication in van der Waals Layered Transition Metal Dichalcogenides. Nat. Commun. 2019, 10 (1), 5488.

(31) Spoor, F. C. M.; Grimaldi, G.; Delerue, C.; Evers, W. H.; Crisp, R. W.; Geiregat, P.; Hens, Z.; Houtepen, A. J.; Siebbeles, L. D. A. Asymmetric Optical Transitions Determine the Onset of Carrier Multiplication in Lead Chalcogenide Quantum Confined and Bulk Crystals. ACS Nano 2018, 12 (5), 4796-4802.

(32) Cirloganu, C. M.; Padilha, L. A.; Lin, Q.; Makarov, N. S.; Velizhanin, K. A.; Luo, H.; Robel, I.; Pietryga, J. M.; Klimov, V. I. Enhanced Carrier Multiplication in Engineered Quasi-Type-II Quantum Dots. Nat. Commun. 2014, 5 (1), 4148.

(33) Guan, L.; Xu, X.; Liang, Y.; Han, S.; Guo, J.; Wang, J.; Li, X. Effect of Atomic Configuration on Band Gap Behaviour in CH3NH3SnxPb1-xI3 Perovskites. Phys. Lett. A 2020, 384 (8), 126173 . 